Harmonic Analysis

\title{
Hardy spaces of differential forms and Riesz transforms on Riemannian manifolds
}

\author{
Pascal Auscher ${ }^{\mathrm{a}}$, Alan McIntosh ${ }^{\mathrm{b}}$, Emmanuel Russ $^{\mathrm{c}}$ \\ a CNRS UMR 8628, université Paris-sud, 91405 Orsay cedex, France \\ ${ }^{\mathrm{b}}$ Centre for Mathematics and its Applications, Mathematical Sciences Institute, Australian National University, Canberra ACT 0200, Australia \\ ${ }^{\mathrm{c}}$ Université Paul-Cézanne LATP, avenue Escadrille Normandie-Niemen, 13397 Marseille cedex 20, France
}

Received 22 August 2006; accepted 14 November 2006

Available online 21 December 2006

Presented by Yves Meyer

\begin{abstract}
Let $M$ be a complete Riemannian manifold. Assuming that the Riemannian measure is doubling, we define, for all $1 \leqslant p \leqslant+\infty$, a Hardy space $H^{p}\left(\Lambda T^{*} M\right)$ of differential forms on $M$, and give two alternative characterizations of $H^{1}\left(\Lambda T^{*} M\right)$. We also prove, for all $1 \leqslant p \leqslant+\infty$, the $H^{p}\left(\Lambda T^{*} M\right)$ boundedness of Riesz transforms on $M$, and show that $H^{p}\left(\Lambda T^{*} M\right)$ has a bounded holomorphic functional calculus. To cite this article: P. Auscher et al., C. R. Acad. Sci. Paris, Ser. I 344 (2007).

(C) 2006 Académie des sciences. Published by Elsevier Masson SAS. All rights reserved.

\section{Résumé}

Espaces de Hardy de formes différentielles et transformées de Riesz sur des variétés riemanniennes. Soit $M$ une variété riemannienne complète. Sous l'hypothèse que la mesure riemannienne est doublante, on définit, pour tout $1 \leqslant p \leqslant+\infty$, un espace de Hardy $H^{p}\left(\Lambda T^{*} M\right)$ de formes différentielles sur $M$, et on donne deux autres caractérisations de $H^{1}\left(\Lambda T^{*} M\right)$. On prouve également, pour tout $1 \leqslant p \leqslant+\infty$, la continuité sur $H^{p}\left(\Lambda T^{*} M\right)$ des transformées de Riesz sur $M$, et on montre que $H^{p}\left(\Lambda T^{*} M\right)$ possède un calcul fonctionnel holomorphe borné. Pour citer cet article : P. Auscher et al., C. R. Acad. Sci. Paris, Ser. I 344 (2007). (C) 2006 Académie des sciences. Published by Elsevier Masson SAS. All rights reserved.
\end{abstract}

\section{Version française abrégée}

Soit $M$ une variété riemannienne complète de dimension $n, \rho$ la distance riemannienne et $d \mu$ la mesure riemannienne. Pour tout $x \in M$ et tout $r>0, B(x, r)$ désigne la boule géodésique ouverte de centre $x$ et de rayon $r$ dont la mesure est notée $V(x, r)$. Pour tout $x \in M$, on note $\Lambda T_{x}^{*} M$ l'algèbre extérieure complexe sur l'espace cotangent $T_{x}^{*} M$. Soit $\Lambda T^{*} M=\bigoplus_{0 \leqslant k \leqslant n} \Lambda^{k} T^{*} M$ le fibré sur $M$ dont la fibre en chaque $x \in M$ est $\Lambda T_{x}^{*} M$ et, pour tout $1 \leqslant p \leqslant+\infty$, $L^{p}\left(\Lambda T^{*} M\right)=\bigoplus_{0 \leqslant k \leqslant n} L^{p}\left(\Lambda^{k} T^{*} M\right)$ l'espace des sections $F$ de $\Lambda T^{*} M$ telles que $|F| \in L^{p}(M, d \mu)$. Soit $d$ la différentielle extérieure, $d^{*}$ son adjoint sur $L^{2}\left(\Lambda T^{*} M\right), D:=d+d^{*}$ l'opérateur de Hodge-Dirac et $\Delta:=D^{2}$ le Laplacien

E-mail addresses: Pascal.Auscher@math.u-psud.fr (P. Auscher), Alan.McIntosh@maths.anu.edu.au (A. McIntosh), emmanuel.russ@univ-cezanne.fr (E. Russ). 
de Hodge-de Rham. Comme $D$ est auto-adjoint, on a la décomposition de $L^{2}\left(\Lambda T^{*} M\right)$ donnée par (4), où, pour tout opérateur linéaire $T$ défini sur un domaine de $L^{2}\left(\Lambda T^{*} M\right), \mathcal{R}(T)$ désigne l'image de $T$ et $\mathcal{N}(T)$ le noyau de $T$. De plus, la somme (4) est topologique.

Si $\theta \in\left(0, \frac{\pi}{2}\right)$, on définit $\Sigma_{\theta}^{0}=\{z \in \mathbb{C} \backslash\{0\} ;|\arg z|<\theta\} \cup\{z \in \mathbb{C} \backslash\{0\} ;|\arg (-z)|<\theta\}$ et on note $H^{\infty}\left(\Sigma_{\theta}^{0}\right)$ l'algèbre des fonctions holomorphes bornées sur $\Sigma_{\theta}^{0}$.

On supposera toujours que $M$ vérifie l'hypothèse (1) sur la croissance du volume des boules. Pour tout $1 \leqslant$ $p<+\infty$, on définit l'espace de Hardy $H^{p}\left(\Lambda T^{*} M\right)$ comme la complétion d'un espace $E^{p}\left(\Lambda T^{*} M\right)$, lui-même défini comme suit. On fixe une fonction $\psi \in H^{\infty}\left(\Sigma_{\theta}^{0}\right)$ telle que $|\psi(z)| \leqslant C \max \left(|z|^{\alpha},|z|^{-\beta}\right)$ pour certains exposants $\alpha, \beta>0$ ne dépendant que de $M$. Alors, si $f$ est une section de $\Lambda T^{*} M, f$ appartient à $E^{p}\left(\Lambda T^{*} M\right)$ si, et seulement si, $f \in \mathcal{R}(D)$ et $(t, x) \mapsto \psi(t D) f(x)$, défini sur $(0,+\infty) \times M$, appartient à l'espace tente $T^{p, 2}\left(\Lambda T^{*} M\right)$ (voir la version anglaise ci-dessous pour la définition précise, et en particulier son indépendence vis-à-vis du choix de $\psi)$. On définit aussi $H^{\infty}\left(\Lambda T^{*} M\right)$ comme le dual de $H^{1}\left(\Lambda T^{*} M\right)$. On a alors le résultat suivant :

\section{Théorème 0.1.}

(i) Pour tout $1 \leqslant p \leqslant+\infty$, la transformée de Riesz $D \Delta^{-1 / 2}$, d'abord définie sur $\mathcal{R}(\Delta)$, se prolonge en un opérateur borné sur $H^{p}\left(\Lambda T^{*} M\right)$. Plus précisément, on a $\left\|D \Delta^{-1 / 2} h\right\|_{H^{p}\left(\Lambda T^{*} M\right)} \sim\|h\|_{H^{p}\left(\Lambda T^{*} M\right)}$.

(ii) Plus généralement, $H^{p}\left(\Lambda T^{*} M\right)$ possède un calcul fonctionnel holomorphe borné, ce qui signifie que, pour tout $1 \leqslant p \leqslant+\infty, f(D)$ est borné sur $H^{p}\left(\Lambda T^{*} M\right)$ pour toute $f \in H^{\infty}\left(\Sigma_{\theta}^{0}\right)$ et on a $\|f(D) h\|_{H^{p}\left(\Lambda T^{*} M\right)} \leqslant$ $C\|f\|_{\infty}\|h\|_{H^{p}\left(\Lambda T^{*} M\right)}$.

L'espace $H^{1}\left(\Lambda T^{*} M\right)$ possède deux autres caractérisations. La première est une décomposition des éléments de $H^{1}\left(\Lambda T^{*} M\right)$ en «molécules», qui généralise la décomposition atomique de $H^{1}\left(\mathbb{R}^{n}\right)$. A la différence des atomes de $H^{1}\left(\mathbb{R}^{n}\right)$, les molécules ne sont pas nécessairement à support dans une boule, mais vérifient une condition de décroissance $L^{2}$.

La deuxième est une caractérisation en termes d'une fonction maximale non tangentielle qui fait intervenir le semigroupe engendré par $\Delta$ et une moyenne $L^{2}$ en espace et en temps (au lieu d'une évaluation ponctuelle comme dans $\mathbb{R}^{n}$ ).

Les énoncés complets et leurs preuves, qui font intervenir des estimations $L^{2}$ hors diagonale de type «Gaffney» [8], ainsi que d'autres résultats, se trouvent dans [2].

\section{Introduction}

Let $M$ be a complete Riemannian manifold with dimension $n, \rho$ the Riemannian distance and $d \mu$ the Riemannian measure. For all $x \in M$ and all $r>0, B(x, r)$ stands for the open geodesic ball with center $x$ and radius $r$, and its measure will be denoted $V(x, r)$. For all $x \in M$, denote by $\Lambda T_{x}^{*} M$ the complex exterior algebra over the cotangent space $T_{x}^{*} M$. Let $\Lambda T^{*} M=\bigoplus_{0 \leqslant k \leqslant n} \Lambda^{k} T^{*} M$ be the bundle over $M$ whose fibre at each $x \in M$ is given by $\Lambda T_{x}^{*} M$ and, for all $1 \leqslant p \leqslant+\infty, L^{p}\left(\Lambda T^{*} M\right)=\bigoplus_{0 \leqslant k \leqslant n} L^{p}\left(\Lambda^{k} T^{*} M\right)$ be the space of sections $F$ of $\Lambda T^{*} M$ such that $|F| \in L^{p}(M, d \mu)$. Denote by $d$ the exterior differentiation and by $\Delta_{\mathrm{LB}}$ the Laplace-Beltrami operator.

It was asked in [12] whether, for $1<p<+\infty$, one has $C_{p}^{-1}\left\|\left(-\Delta_{\mathrm{LB}}\right)^{1 / 2} f\right\|_{L^{p}(M)} \leqslant\|d f\|_{L^{p}\left(\Lambda^{1} T^{*} M\right)} \leqslant$ $C_{p}\left\|\left(-\Delta_{\mathrm{LB}}\right)^{1 / 2} f\right\|_{L^{p}(M)}$ for suitable functions $f$. Both of these two inequalities were investigated in several papers and established for some ranges of $p$ 's under various geometric assumptions on $M$ (see $[1,3,7]$ ). These inequalities break down for $p=1$ (even in $\mathbb{R}^{n}$ ), but some endpoint results, involving the Hardy space $H^{1}(M)$ (which is a strict subspace of $\left.L^{1}(M)\right)$, can still be given. More precisely, say that $M$ satisfies the doubling property if

$$
\exists C>0 \forall x \in M \forall r>0 \quad V(x, 2 r) \leqslant C V(x, r) .
$$

Assumption (1) means that $(M, \rho, d \mu)$ is a space of homogeneous type, and $H^{1}(M)$ in this context is the CoifmanWeiss Hardy space (see [6]). Say that $M$ satisfies the $L^{2}$-Poincaré inequality for balls if

$$
\exists C>0 \forall x_{0} \in M \forall r>0 \forall f \in C^{\infty}\left(B\left(x_{0}, 2 r\right)\right) \int_{B\left(x_{0}, r\right)}\left|f(x)-f_{B\left(x_{0}, r\right)}\right|^{2} d x \leqslant C r^{2} \int_{B\left(x_{0}, 2 r\right)}|d f(x)|^{2} d x,
$$


where $f_{B\left(x_{0}, r\right)}$ denotes the mean-value of $f$ on $B\left(x_{0}, r\right)$. It was proved in [11] that, if $M$ satisfies (1) and (2), then there exists $C>0$ such that, for all $f \in H^{1}(M)$,

$$
\left\|d\left(-\Delta_{\mathrm{LB}}\right)^{-1 / 2} f\right\|_{L^{1}\left(\Lambda^{1} T^{*} M\right)} \leqslant C\|f\|_{H^{1}(M)} .
$$

In other words, the Riesz transform $R:=d\left(-\Delta_{\mathrm{LB}}\right)^{-1 / 2}$ is $H^{1}(M)-L^{1}\left(\Lambda^{1} T^{*} M\right)$ bounded. Note that Gaussian pointwise estimates for the kernel of the semigroup generated by $-\Delta_{\mathrm{LB}}$, which hold under assumptions (1) and (2), are an essential tool of the proof. In the present Note, we define Hardy spaces of differential forms (already considered in the Euclidean context in [10]) which allow us, in particular, to obtain an $H^{1}-H^{1}$ boundedness result for the Riesz transform under the only assumption (1) (i.e. even when the manifold does not satisfy (2)). Moreover, this $H^{1}$-boundedness of the Riesz transform will hold for general differential forms, and not only for functions.

\section{Hardy spaces and Riesz transforms}

\subsection{Definition of Hardy spaces}

In the Euclidean context, Hardy spaces are closely related to tent spaces, introduced in [5]. Indeed, a measurable function $f$ belongs to $H^{1}\left(\mathbb{R}^{n}\right)$ if, and only if, $t(-\Delta)^{1 / 2} \mathrm{e}^{-t(-\Delta)^{1 / 2}} f$ belongs to the tent space $T^{1,2}\left(\mathbb{R}^{n}\right)$ (and $t(-\Delta)^{1 / 2} \mathrm{e}^{-t(-\Delta)^{1 / 2}}$ may be replaced by more general functions of $\left.\Delta\right)$. This connection provides alternative proofs of the $H^{1}\left(\mathbb{R}^{n}\right)-B M O\left(\mathbb{R}^{n}\right)$ duality and of the atomic decomposition for functions in $H^{1}\left(\mathbb{R}^{n}\right)$. It turns out that this point of view on Hardy spaces can be generalized to our context.

From now on, we always assume that $M$ is a complete Riemannian manifold satisfying (1). Denote by $d^{*}$ the adjoint of $d$ in $L^{2}\left(\Lambda T^{*} M\right)$, by $D:=d+d^{*}$ the Hodge-Dirac operator on $M$ and by $\Delta:=D^{2}$ the Hodge-de Rham Laplacian. We remark that, owing to the self-adjointness of $D$, one has

$$
L^{2}\left(\Lambda T^{*} M\right)=\overline{\mathcal{R}(D)} \oplus \mathcal{N}(D),
$$

where $\mathcal{R}(T)$ denotes the range of $T$ and $\mathcal{N}(T)$ the null-space of $T$ in $L^{2}\left(\Lambda T^{*} M\right)$, and the sum is topological.

Since we deal with general forms (and not only with functions), Gaussian pointwise estimates for the kernel of the semigroup generated by $\Delta$ are not available. However, weaker estimates, namely $L^{2}$ off-diagonal estimates (in the spirit of Gaffney, [8]), hold for this semigroup in any complete Riemannian manifold, and we prove that these estimates are sufficient to develop a theory of Hardy spaces of differential forms under the only assumption (1).

Let us now define tent spaces on $M$. For all $x \in M$, let $\Gamma(x)=\{(t, y) \in M \times(0,+\infty) ; d(y, x)<t\}$ be the cone with vertex $x$ and aperture 1 . If $\left(F_{t}\right)_{t>0}$ is a family of measurable sections of $\Lambda T^{*} M$ such that $F(t, y):=F_{t}(y)$ is measurable on $(0,+\infty) \times M$, set, for all $x \in M$,

$$
\mathcal{A} F(x)=\left(\iint_{\Gamma(x)} \frac{|F(t, y)|^{2}}{V(x, t)} d y \frac{d t}{t}\right)^{1 / 2}
$$

(here and after, we write $d x, d y, \ldots$ instead of $d \mu(x), d \mu(y), \ldots)$. If $1 \leqslant p<+\infty$, say that $F \in T^{p, 2}\left(\Lambda T^{*} M\right)$ if and only if $\|F\|_{T^{p, 2}\left(\Lambda T^{*} M\right)}:=\|\mathcal{A} F\|_{L^{p}(M)}<+\infty$.

The definition of Hardy spaces also involves two operators built upon $D$. If $\theta \in\left(0, \frac{\pi}{2}\right)$, set $\Sigma_{\theta}^{0}=\{z \in \mathbb{C} \backslash\{0\}$; $|\arg z|<\theta\} \cup\{z \in \mathbb{C} \backslash\{0\} ;|\arg (-z)|<\theta\}$ and denote by $H^{\infty}\left(\Sigma_{\theta}^{0}\right)$ the algebra of bounded holomorphic functions on $\Sigma_{\theta}^{0}$. Since the operator $D$ is self-adjoint in $L^{2}\left(\Lambda T^{*} M\right)$, one may define the $L^{2}$-bounded operator $f(D)$ for any $f \in H^{\infty}\left(\Sigma_{\theta}^{0}\right)$.

Given $\sigma, \tau>0$, define $\Psi_{\sigma, \tau}\left(\Sigma_{\theta}^{0}\right)$ to be the set of holomorphic functions $\psi \in H^{\infty}\left(\Sigma_{\theta}^{0}\right)$ which satisfy $|\psi(z)| \leqslant C$ $\inf \left\{|z|^{\sigma},|z|^{-\tau}\right\}$ for some $C>0$ and all $z \in \Sigma_{\theta}^{0}$, and set $\Psi\left(\Sigma_{\theta}^{0}\right)=\bigcup_{\sigma, \tau>0} \Psi_{\sigma, \tau}\left(\Sigma_{\theta}^{0}\right)$. Given $\psi \in \Psi\left(\Sigma_{\theta}^{0}\right)$ for some $\theta>0$, set $\psi_{t}(z)=\psi(t z)$ for all $t>0$ and all $z \in \Sigma_{\theta}^{0}$ and define, for all $h \in L^{2}\left(\Lambda T^{*} M\right)$, all $H \in T^{2,2}\left(\Lambda T^{*} M\right)$ and all $t>0$,

$$
\left(\mathcal{Q}_{\psi} h\right)_{t}=\psi_{t}(D) h \quad \text { and } \quad \mathcal{S}_{\psi} H=\int_{0}^{+\infty} \psi_{t}(D) H_{t} \frac{d t}{t},
$$


where the integral converges in the $L^{2}\left(\Lambda T^{*} M\right)$ strong topology. It follows from the spectral theorem that $\mathcal{Q}_{\psi}$ is $L^{2}\left(\Lambda T^{*} M\right)-T^{2,2}\left(\Lambda T^{*} M\right)$ bounded and $\mathcal{S}_{\psi}$ is $T^{2,2}\left(\Lambda T^{*} M\right)-L^{2}\left(\Lambda T^{*} M\right)$ bounded.

Definition of $H^{p}\left(\Lambda T^{*} M\right)$ for $1 \leqslant p \leqslant 2$. Fix a function $\psi \in \Psi_{\beta, 2}\left(\Sigma_{0}^{\theta}\right)$ (where $\beta$ is a positive number only depending on $M$ ) and set $E_{\psi}^{p}\left(\Lambda T^{*} M\right):=\mathcal{S}_{\psi}\left(T^{p, 2}\left(\Lambda T^{*} M\right) \cap T^{2,2}\left(\Lambda T^{*} M\right)\right)$ equipped with the semi-norm

$$
\|h\|_{H_{\psi}^{p}\left(\Lambda T^{*} M\right)}=\inf \left\{\|H\|_{T^{p, 2}\left(\Lambda T^{*} M\right)} ; H \in T^{p, 2}\left(\Lambda T^{*} M\right) \cap T^{2,2}\left(\Lambda T^{*} M\right), \mathcal{S}_{\psi} H=h\right\} .
$$

It turns out that $E_{\psi}^{p}\left(\Lambda T^{*} M\right)$ is independent of $\psi$ (provided that $\psi \in \Psi_{\beta, 2}\left(\Sigma_{0}^{\theta}\right)$ ) and $\|\cdot\|_{H_{\psi}^{p}\left(\Lambda T^{*} M\right)}$ is actually a norm. Finally, if $\tilde{\psi} \in \Psi_{1, \beta+1}\left(\Sigma_{0}^{\theta}\right)$, one has $E_{\psi}^{p}\left(\Lambda T^{*} M\right)=\left\{h \in \mathcal{R}(D) ; \mathcal{Q}_{\tilde{\psi}} h \in T^{p, 2}\left(\Lambda T^{*} M\right)\right\}$ and $\|h\|_{H_{\psi}^{p}\left(\Lambda T^{*} M\right)} \sim$ $\left\|\mathcal{Q}_{\tilde{\psi}} h\right\|_{T^{p, 2}\left(\Lambda T^{*} M\right)}$. We can then define $H_{\psi}^{p}\left(\Lambda T^{*} M\right)$ as the completion of $E_{\psi}^{p}\left(\Lambda T^{*} M\right)$ under any of the equivalent norms $\|\cdot\|_{E_{\psi}^{p}\left(\Lambda T^{*} M\right)}$, and write $H^{p}\left(\Lambda T^{*} M\right)$ instead of $H_{\psi}^{p}\left(\Lambda T^{*} M\right)$.

Definition of $H^{p}\left(\Lambda T^{*} M\right)$ for $2 \leqslant p<+\infty$. If $\psi \in \Psi_{1, \beta+1}\left(\Sigma_{0}^{\theta}\right)$, define $E_{\psi}^{p}\left(\Lambda T^{*} M\right)$ and the corresponding seminorm as before. Similarly, this definition does not depend on the choice of $\psi \in \Psi_{1, \beta+1}\left(\Sigma_{0}^{\theta}\right)$, the semi-norm is a norm and, for all $\tilde{\psi} \in \Psi_{\beta, 1}\left(\Sigma_{0}^{\theta}\right), h \in E_{\psi}^{p}\left(\Lambda T^{*} M\right)$ if and only if $h \in \mathcal{R}(D)$ and $\mathcal{Q}_{\tilde{\psi}} h \in T^{p, 2}\left(\Lambda T^{*} M\right)$, and one has $\|h\|_{H_{\psi}^{p}\left(\Lambda T^{*} M\right)} \sim\left\|\mathcal{Q}_{\tilde{\psi}} h\right\|_{T^{p, 2}\left(\Lambda T^{*} M\right)}$. We define $H_{\psi}^{p}\left(\Lambda T^{*} M\right)$ as the completion of $E_{\psi}^{p}\left(\Lambda T^{*} M\right)$ under any of the previous equivalent norms, and since this space does not depend on $\psi$, it will be denoted by $H^{p}\left(\Lambda T^{*} M\right)$.

Finally, define $H^{\infty}\left(\Lambda T^{*} M\right)$ as the dual space of $H^{1}\left(\Lambda T^{*} M\right)$.

One has the following duality and interpolation results for Hardy spaces:

Theorem 2.1. For all $1 \leqslant p<+\infty$, the dual of $H^{p}\left(\Lambda T^{*} M\right)$ is isomorphic to $H^{p^{\prime}}\left(\Lambda T^{*} M\right)$, with $1 / p+1 / p^{\prime}=1$.

Theorem 2.2. Let $1 \leqslant p_{0}<p<p_{1} \leqslant+\infty$ and $\theta \in(0,1)$ such that $1 / p=(1-\theta) / p_{0}+\theta / p_{1}$. Then

$$
\left[H^{p_{0}}\left(\Lambda T^{*} M\right), H^{p_{1}}\left(\Lambda T^{*} M\right)\right]_{\theta}=H^{p}\left(\Lambda T^{*} M\right) .
$$

For all $1 \leqslant p<+\infty$, one naturally defines

$$
H_{d}^{p}\left(\Lambda T^{*} M\right)=\overline{\mathcal{R}(d) \cap H^{p}\left(\Lambda T^{*} M\right)} \quad \text { and } \quad H_{d^{*}}^{p}\left(\Lambda T^{*} M\right)=\overline{\mathcal{R}\left(d^{*}\right) \cap H^{p}\left(\Lambda T^{*} M\right)},
$$

where the closure is taken in the $H^{p}\left(\Lambda T^{*} M\right)$ topology. The splitting $\Lambda T^{*} M=\bigoplus_{0 \leqslant k \leqslant n} \Lambda^{k} T^{*} M$ also allows us to define $H^{p}\left(\Lambda^{k} T^{*} M\right), H_{d}^{p}\left(\Lambda^{k} T^{*} M\right)$ and $H_{d^{*}}^{p}\left(\Lambda^{k} T^{*} M\right)$ for all $0 \leqslant k \leqslant n$.

\subsection{Riesz transforms and bounded holomorphic functional calculus}

Define the Riesz transform as the operator $D \Delta^{-1 / 2}$ on $\mathcal{R}(\Delta)$. Then, one has:

Theorem 2.3. For all $1 \leqslant p \leqslant+\infty$, the Riesz transform $D \Delta^{-1 / 2}$ extends to an $H^{p}\left(\Lambda T^{*} M\right)$-bounded operator. More precisely, one has $\left\|D \Delta^{-1 / 2} h\right\|_{H^{p}\left(\Lambda T^{*} M\right)} \sim\|h\|_{H^{p}\left(\Lambda T^{*} M\right)}$.

More generally, $H^{p}\left(\Lambda T^{*} M\right)$ has a bounded holomorphic functional calculus:

Theorem 2.4. For all $1 \leqslant p \leqslant+\infty, f(D)$ is $H^{p}\left(\Lambda T^{*} M\right)$-bounded for all $f \in H^{\infty}\left(\Sigma_{\theta}^{0}\right)$ with $\|f(D) h\|_{H^{p}\left(\Lambda T^{*} M\right)} \leqslant$ $C\|f\|_{\infty}\|h\|_{H^{p}\left(\Lambda T^{*} M\right)}$.

As a consequence of Theorem 2.3, one has the following Hodge decomposition for $H^{p}\left(\Lambda T^{*} M\right)$ if $1 \leqslant p<+\infty$ :

Theorem 2.5. For all $1 \leqslant p<+\infty$, one has $H^{p}\left(\Lambda T^{*} M\right)=H_{d}^{p}\left(\Lambda T^{*} M\right) \oplus H_{d^{*}}^{p}\left(\Lambda T^{*} M\right)$, and the sum is topological. 


\section{Two equivalent definitions of $H^{1}\left(\Lambda T^{*} M\right)$}

\subsection{The decomposition into molecules}

It is well known that $H^{1}\left(\mathbb{R}^{n}\right)$ has an atomic decomposition [4,9]. Here, we show that any element of $H^{1}\left(\Lambda T^{*} M\right)$ can be decomposed in a series of 'molecules'.

Fix $C>0$. If $B \subset M$ is a ball with radius $r$ and if $\left(\chi_{k}\right)_{k} \geqslant 0$ is a sequence of nonnegative $C^{\infty}$ functions on $M$ with bounded support, say that $\left(\chi_{k}\right)_{k} \geqslant 0$ is adapted to $B$ if $\chi_{0}$ is supported in $4 B, \chi_{k}$ is supported in $2^{k+2} B \backslash 2^{k-1} B$ and $\left\|\left|d \chi_{k}\right|\right\|_{\infty} \leqslant \frac{C}{2^{k} r}$ for all $k \geqslant 1$, and $\sum_{k \geqslant 0} \chi_{k}=1$ on $M$. Note that, when $C>0$ is large enough, there exist sequences adapted to any fixed ball.

Let $N$ be a positive integer. If $a \in L^{2}\left(\Lambda T^{*} M\right), a$ is called an $N$-molecule if and only if there exists a ball $B \subset M$ with radius $r$, a section $b \in L^{2}\left(\Lambda T^{*} M\right)$ such that $a=D^{N} b$, and a sequence $\left(\chi_{k}\right)_{k \geqslant 0}$ adapted to $B$ such that, for all $k \geqslant 0$,

$$
\left\|\chi_{k} a\right\|_{L^{2}\left(\Lambda T^{*} M\right)} \leqslant 2^{-k} V^{-1 / 2}\left(2^{k} B\right) \text { and }\left\|\chi_{k} b\right\|_{L^{2}\left(\Lambda T^{*} M\right)} \leqslant 2^{-k} r^{N} V^{-1 / 2}\left(2^{k} B\right) .
$$

The decomposition of $H^{1}\left(\Lambda T^{*} M\right)$ into molecules can be stated as follows:

Theorem 3.1. There exists an integer $N_{0} \geqslant 1$ only depending on $M$ such that, for any integer $N \geqslant N_{0}$ and any section $f$ of $\Lambda T^{*} M, f \in H^{1}\left(\Lambda T^{*} M\right)$ if and only if there exist a sequence of $N$-molecules $\left(a_{j}\right)_{j} \geqslant 1$ and a sequence $\left(\lambda_{j}\right)_{j \geqslant 1} \in l^{1}$ such that $f=\sum_{j \geqslant 1} \lambda_{j} a_{j}$. Moreover, $\|f\|_{H^{1}\left(\Lambda T^{*} M\right)} \sim \inf \sum_{j \geqslant 1}\left|\lambda_{j}\right|$, where the infimum is taken over all such decompositions of $f$.

\subsection{The maximal characterization}

As in the case of $H^{1}\left(\mathbb{R}^{n}\right)$, the $H^{1}\left(\Lambda T^{*} M\right)$ space admits a characterization in terms of a non-tangential maximal function of the semigroup generated by $\Delta$, but here, the usual pointwise evaluation has to be replaced by an $L^{2}$ mean value because of the lack of pointwise kernel estimates of the semigroup. For all $x \in M$ and all $0<r<t$, set $B((x, t), r)=B(x, r) \times(t-r, t+r)$. If $f \in L^{2}\left(\Lambda T^{*} M\right)$ and $x \in M$, define

$$
f^{*}(x)=\sup _{(t, y) \in \Gamma(x)}\left(\frac{1}{t V(y, t)} \iint_{B\left((y, t), \frac{t}{4}\right)}\left|\mathrm{e}^{-s^{2} \Delta} f(z)\right|^{2} d z d s\right)^{1 / 2} .
$$

Define $H_{\max }^{1}\left(\Lambda T^{*} M\right)$ as the completion of $\left\{f \in \mathcal{R}(D) ; f^{*} \in L^{1}(M)\right\}$ for that norm and set $\|f\|_{H_{\max }^{1}\left(\Lambda T^{*} M\right)}:=$ $\left\|f^{*}\right\|_{L^{1}(M)}$. Then, the following characterization of $H^{1}\left(\Lambda T^{*} M\right)$ holds:

Theorem 3.2. One has $H_{\max }^{1}\left(\Lambda T^{*} M\right)=H^{1}\left(\Lambda T^{*} M\right)$.

\section{Comparisons with other functional spaces}

For all $1 \leqslant p<+\infty$, one can compare $H^{p}\left(\Lambda T^{*} M\right)$ and $L^{p}\left(\Lambda T^{*} M\right)$ :

Theorem 4.1. The following inclusions are valid:

(a) for $1 \leqslant p \leqslant 2, H^{p}\left(\Lambda T^{*} M\right) \subset \overline{\mathcal{R}(D) \cap L^{p}\left(\Lambda T^{*} M\right)} L^{p}\left(\Lambda T^{*} M\right)$,

(b) for $2 \leqslant p<+\infty, \overline{\mathcal{R}(D) \cap L^{p}\left(\Lambda T^{*} M\right)} L^{L^{p}\left(\Lambda T^{*} M\right)} \subset H^{p}\left(\Lambda T^{*} M\right)$.

Specializing to the case of functions, i.e. 0-forms, one can also compare the usual $H^{1}(M)$ space (i.e. the CoifmanWeiss Hardy space) with $H_{d^{*}}^{1}\left(\Lambda^{0} T^{*} M\right)$ :

Theorem 4.2. Under assumption (1), one has $H_{d^{*}}^{1}\left(\Lambda^{0} T^{*} M\right) \subset H^{1}(M)$ and the inclusion is strict in general. If one assumes furthermore that $M$ satisfies (2), then $H_{d^{*}}^{1}\left(\Lambda^{0} T^{*} M\right)=H^{1}(M)$. 
The proofs of all the previous theorems, as well as further results, can be found in [2]. This work was supported by the bilateral CNRS/ARC linkage 2003/2004, Espaces de Hardy de formes différentielles/Hardy spaces of differential forms.

\section{References}

[1] P. Auscher, T. Coulhon, X.T. Duong, S. Hofmann, Riesz transforms on manifolds and heat kernel regularity, Ann. Sci. École Norm. Sup. 37 (6) (2004) 911-957.

[2] P. Auscher, A. McIntosh, E. Russ, Hardy spaces of differential forms on Riemannian manifolds, http://arxiv.org/abs/math.DG/0611334.

[3] D. Bakry, Etude des transformations de Riesz dans les variétés riemanniennes à courbure de Ricci minorée, in: Séminaire de Probabilités, XXI, in: Lecture Notes in Math., vol. 1247, Springer, Berlin, 1987, pp. 137-172.

[4] R. Coifman, A real-variable characterization of $H^{p}$, Studia Math. 51 (1974) 269-274.

[5] R. Coifman, Y. Meyer, E.M. Stein, Some new function spaces and their applications to harmonic analysis, J. Funct. Anal. 62 (1985) $304-335$.

[6] R. Coifman, G. Weiss, Extensions of Hardy spaces and their use in analysis, Bull. Amer. Math. Soc. 83 (1977) $569-645$.

[7] T. Coulhon, X.T. Duong, Riesz transforms for $1 \leqslant p \leqslant 2$, Trans. Amer. Math. Soc. 351 (3) (1999) 1151-1169.

[8] M.P. Gaffney, The conservation property of the heat equation on Riemannian manifolds, Comm. Pure Appl. Math. 12 (1959) 1-11.

[9] R.H. Latter, A characterization of $H^{p}\left(\mathbb{R}^{n}\right)$ in terms of atoms, Studia Math. 62 (1) (1978) 93-101.

[10] Z. Lou, A. McIntosh, Hardy spaces of exact forms on $\mathbb{R}^{n}$, Trans. Amer. Math. Soc. 357 (4) (2005) 1469-1496.

[11] E. Russ, $H^{1}-L^{1}$ boundedness of Riesz transforms on Riemannian manifolds and on graphs, Potential Anal. 14 (2001) 301-330.

[12] R.S. Strichartz, Analysis of the Laplacian on the complete Riemannian manifold, J. Funct. Anal. 52 (1) (1983) 48-79. 